\title{
Budesonide-Hydroxypropyl- $\beta$-Cyclodextrin Inclusion Complex in Poloxamer 407 and Poloxamer 407/403Systems: A Structural Study by Small Angle X-Ray Scattering (SAXS)
}

\author{
Margareth KKD Franco*1, Melissa I Alkschbirs ${ }^{2}$, Alessandra CS Akkari ${ }^{4}$, Fabiano Yokaichiya ${ }^{3}$ and Daniele Ribeiro de \\ Araujo $^{4}$
}

${ }^{1}$ Instituto de Pesquisas Energéticas e Nucleares-IPEN, São Paulo/Brazil

${ }^{2}$ Universidade Estadual de Campinas-UNICAMP, Campinas/Brazil

${ }^{3}$ Helmholtz-Zentrum Berlin, Berlin/Germany

${ }^{4}$ Universidade Federal do ABC, São André/Brazil

Received: 制: October 22, 2018; Published: 盋: November 02, 2018

*Corresponding author: Margareth KKD Franco, Instituto de Pesquisas Energéticas e Nucleares - IPEN, Brazil

\begin{abstract}
Poloxamer (PL), copolymers composed of polyethylene oxide (PEO) and polypropylene oxide (PPO) units, have been studied for several applications considering them as tensoatives, stabilizers, solubilizers and nano carriers. For pharmaceutical applications, they are of great interest for the development of drug-delivery systems as unique or binary systems (association of two types of PL) and for forming supramolecular complexes with other molecules (i.e. 2-hydroxypropyl- $\beta$-cyclodextrin-HP- $\beta$-CD), especially for poorly soluble drugs, such as budesonide (BUD), a glucocorticoid widely used for the treatment of ulcerative colitis. This study reports the structural studies, by Small Angle X-ray Scattering (SAXS), looking forward the understanding of the mechanisms and models of interaction between BUD, HP- $\beta$-CD or BUD-HP- $\beta$-CD inclusion complex and PL407 or its binary system, PL407-PL403. The SAXS analysis revealed the systems organization in a lamellar structure, for PL407 and PL407-PL403, even after addition of HP- $\beta-C D$, BUD or the BUD-HP- $\beta-C D$ inclusion complex. Those differences are results of typical interactions between BUD or HP- $\beta$-CD with the micellar core (PPO region), while the presence of inclusion complex is observed on the micellar hydrated region (PEO). Those results are of great interest on the study of physico-chemical aspects applied to the development of drug-delivery formulations.
\end{abstract}

Keywords: Small Angle X-ray Scattering; cyclodextrins inclusion complex; supramolecular structure; copolymer

Abbreviations: PL: Poloxamer; PEO: Poly Ethylene Oxide; PPO: Poly Propylene Oxide; BUD: Budesonide; SAXS: Small Angle X-ray Scattering; UC: Ulcerative Colitis; CD: Cyclodextrins

\section{Introduction}

Systemic glucocorticoids have been traditionally used to induce remission in patients with inflammatory bowel disease such as ulcerative colitis (UC), due to their potent anti-inflammatory effects. However, the wide range of adverse effects associated with these drugs stimulated the development of new agents with safer pharmacological profile and predominant local anti-inflammatory activity, such as budesonide (BUD) [1,2]. Cyclodextrins (CD) have been used as adjuvants in different pharmaceutical formulations due to their ability to form of inclusion complexes. In special, the $\beta$-CD derivative, 2 -hydroxypropyl- $\beta$-cyclodextrin (HP- $\beta$-CD), is an effective alternative for complexation with poorly soluble drugs
$[3,4]$ such as BUD. For pharmaceutical applications, it has been reported the development of ternary complexes, i.e., comprising three different molecular entities. Two of those compounds refer to the drug and $C D$, while the third one may have different origins and purposes, such as natural or synthetic polymers [5]. In addition to $\mathrm{CD}$, polymers such as poloxamers (PL) improve physico-chemical properties of the drug, as well as the entrapment and the efficiency of release, also optimizing the cost, toxicity and the production of the scaled-up pharmaceutical formulations [4].

Poloxamers (PL) are triblock copolymers composed of polyethylene oxide (PEO) and polypropylene oxide (PPO) units 
organized as PEO-PPO-PEO structure. The numbers of PEO and PPO units as well as the PEO: PPO ratio determine their structural and physico-chemical features (molecular weight, hydrophiliclipophilic balance-HLB and critical micellar concentration-CMC). Due to their amphiphilic characteristics, PL are able to selfassembly in micelles (when in aqueous solution above their CMC) and also are thermoresponsive, as a result of the PPO dehydration groups and micellar aggregation, forming hydrogels at a critical gelation temperature (CGT). Those properties allow the PL application in the chemical, cosmetic and pharmaceutical fields [6]. Among glucocorticoid drugs, BUD presents low aqueous solubility and extensive first-pass biotransformation which are limitations for administration by different routes. In order to develop new pharmaceutical formulations able to increase the aqueous solubility and to maintain the drug into the site of administration (specially into the colonic region), in situ forming hydrogels have been proposed as promising alternatives.

In fact, different components (drugs, tensoactives, organic solvents, solubilizers) incorporated into PL-based formulations have directed their investigations [7-11]. Then, to reach those goals, we present the structural effects of the incorporation of BUD-HP- $\beta$ CD inclusion complex into the PL-based thermoreversible hydrogels composed of PL 407 and PL 403, with hydrophilic lipophilic balances (HLB) of 22 and 8, respectively. The formation of binary systems composed of hydrophilic (PL407) and hydrophobic PL copolymers, can modulate physico-chemical and biopharmaceutical properties (such as drug solubility and release kinetics) [12]. Some reports have presented specific structural studies about the interaction between CD and PL [13-19]. However, none of them considered the possible interactions between drug: $\mathrm{CD}$ inclusion complexes and PL-based binary systems with different hydrophilic-lipophilic balances, such as PL407-PL403. Then, in this article, we reported the effects of HP- $\beta$-CD, BUD or BUD-HP- $\beta$-CD inclusion complex incorporation into PL 407 or PL407-PL403 hydrogels, determined by Small Angle X-ray Scattering (SAXS) analysis, in order to study the PL binary system phase organization mechanism and its influence on the supramolecular structure of PL-based hydrogels as well as to elaborate their association model.

\section{Materials and Methods}

Budesonide (BUD), 2-Hydroxypropyl- $\beta$-cyclodextrin (HP$\beta$-CD), Poloxamer 403 (Pluronic $\AA$ P-123) and Poloxamer 407 (Pluronic ${ }^{\circledR}$ F-127) were purchased from Sigma-Aldrich Chem. Co., NO, USA. All chemicals and solvents were of analytical grade. In order to obtain the BUD: HP- $\beta$-CD inclusion complex, HP- $\beta$ $\mathrm{CD}$ and BUD were mixed in water, at 1:1 drug: CD molar ratio, homogenized for $24 \mathrm{~h}$ (at $25^{\circ} \mathrm{C}$ ), lyophilized and stored at $-20{ }^{\circ} \mathrm{C}$ until use or incorporation into the PL-based systems. After that, PL-based formulations were obtained by PL 407 (18 or $20 \%$ m/v) isolated or in association with PL403 (at $2 \% \mathrm{~m} / \mathrm{v}$ ), in water, and kept at cold bath under magnetic stirring (100 rpm) until complete polymer dissolution. BUD was incorporated into the PL-systems at $0.5 \mathrm{mg} / \mathrm{mL}$ and BUD: HP- $\beta$-CD inclusion complex or HP- $\beta$-CD were added to the different PL-formulations for obtaining the same final BUD concentration [12]. It is noteworthy that the CD-PL systems were used in this study as a comparison parameter (control) to the inclusion complex (CPX)-PL.

Morphological observations were performed using small angle X-ray scattering (SAXS). The experiments were carried out at the D11A-SAXS1 beamline at the Brazilian Synchrotron Light Laboratory (LNLS) in Brazil. A parallel X-ray beam irradiates a cylindrical sample holder. The radiation wavelength was $1.55 \AA$ and a Pilatus detector at $936 \mathrm{~mm}$ distance was used to give a scattering vector $(q=4 \pi / \lambda \sin (\theta))$, where $2 \theta$ is the scattering angle ranging from 0.06 to $2.00 \mathrm{~nm}^{-1}$. Water scattering curves were collected and subtracted from the samples scattering curve, taking into account its attenuation. All the measurements were performed at $25^{\circ} \mathrm{C}$.

\section{Results and Discussion}

Figure 1 shows the comparison of SAXS measurements for the PL407 systems at $18 \%$ (1a), $20 \%$ (1b) and its binary system at 18-2\% PL407-PL403 (3c). In general, the SAXS profiles showed a lamellar phase organization, with spacing $d=\frac{2 \Pi}{q^{*}} \square 18 n \mathrm{~m}$, even after insertion of BUD, HP- $\beta$-CD or their inclusion complex. In this context, reports in the literature have shown that PL407 units (PPO units) interact with the hydrophobic cavity of HP- $\beta$-CD and displace guest molecules, such as drugs, leading to the formation of PL: CD non-covalent complexes, named polypseudo rotaxanes [16,2022]. Although it is known that beta CD can disturb the poloxamers micellar structure by reducing the critical micellar concentration since it makes them more hydrophilic [23], this effect is not expected for the system studied here since the CD:PL molar ration were lower $(\sim 1: 10)$ compared to the literature (5:1) [22] and the PL407 and PL403 molecules have long PPG chains. However, after insertion of BUD-HP- $\beta$-CD complex into the PL-systems this phenomenon was not observed, since the high association constant $\left(\mathrm{K}_{\mathrm{a}}\right)$ value between BUD and HP- $\beta$-CD $\left(\mathrm{K}_{\mathrm{a}}=8662.8 \mathrm{M}^{-1}\right)$ [12] reduces the possibility of the drug displacement from the HP- $\beta-C D$, allowing the accommodation of the inclusion complex entities into the lamellar PEO region.

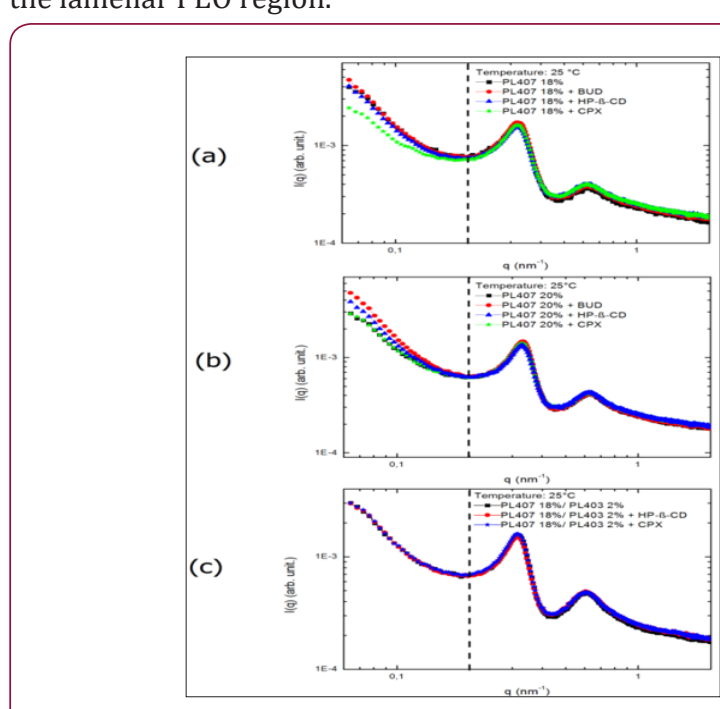

Figure 1: SAXS measurements for (a) $18 \%$ PL407; (b) $20 \%$ PL407 and (c) 18-2 \% PL407-PL403 isolated and containing BUD, hydroxypropyl- $\beta$-cyclodextrin $(\mathrm{HP}-\beta-\mathrm{CD})$ or inclusion complex (CPX). 
In order to fit the SAXS data for low q, as shown in Figure 2, we apply the model of a modified Ornstein-Zernike form, as used in the literature [24-26] to describe I(q):

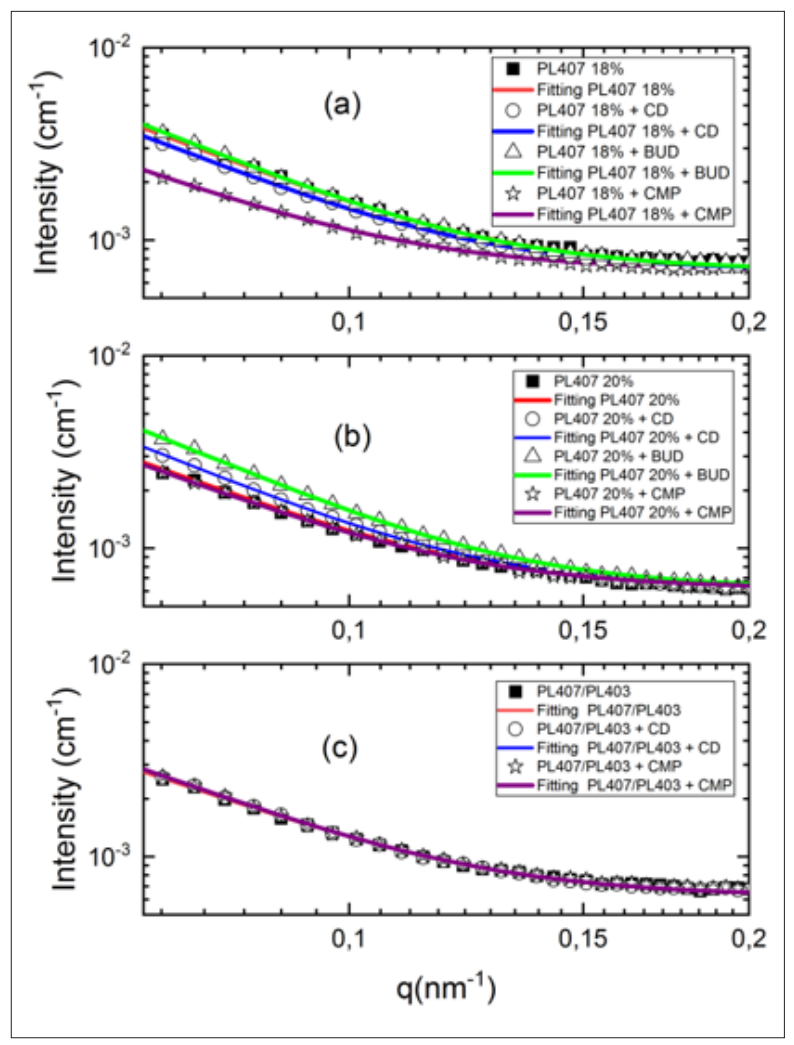

Figure 2: Fitting of SAXS curves using a modified Ornstein-Zernike form for (a) PL 407 18\%; (b) PL407 20\% and (c) PL407 18\%/PL 403 2\% isolated and containing budesonide (BUD), 2-hydroxypropyl- $\beta$-cyclodextrin (HP$\beta-\mathrm{CD})$ and the complex (CMP): budesonide-2hydroxypropyl- $\beta$-cyclodextrin inclusion complex.

$$
I(q)=I(0) \cdot \frac{1}{\left(1+\beta^{2} q^{2}\right)^{\alpha}} \cdot \frac{1}{\left(1+\delta^{2} q^{2}\right)^{\eta}}
$$

In this model we have four free parameters in addition to the amplitude $I(0): \beta$ is associated to the cluster size (Figure 3 ), $\delta$ is related to the primary-particle size, $\alpha$ is the mass fractal dimension, $\eta$ is the surface fractal dimension. The values of $\beta$ (cluster size) and $\delta$ (primary-particle size) for all the systems, are shown in the Table 1 . The values of the mass fractal dimension, $\alpha$, for all the systems are the same, 0.69 and the surface fractal dimension, $\eta$, is 2.32 . Finally, other important point to be considered is regarding to PL final concentration into the systems, resulting in different CD:PL molar ratio, being 1:12 and 1:14 for PL407 systems at $18 \%$ or 20 $\%$, respectively. In fact, different CD:PL ratios influenced on the accommodation of HP- $\beta$-CD into the lamellae evoking pronouncing changes in the primary-particle size, as observed in the Table 1 , for all the concentrations of PL 407, due to the interaction and aggregation of PL unimers at higher concentrations. However, for the binary system the insertion of BUD, HP- $\beta$-CD or BUD-HP$\beta$-CD inclusion complex did not evoke an evident change on the primary-particle size (Table 1). This observation is resulting of the presence of PL403 into the systems (1:4 PL403:PL407 molar ratio), since its low molecular weight $(M W=5800)$ and shorter number of PEO units (PEO units = 39.2), compared to PL407 (MW = 12600 and PEO units $=200.5$ ) [23], allowed the accommodation of those components into the lamellae.

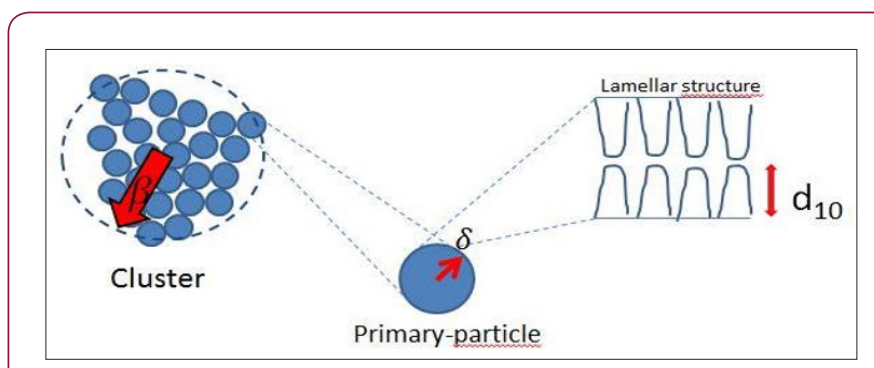

Figure 3: Schematic model proposed for the cluster size, the primary particle and the lamellar structure of the systems discussed in this study. (d10 is interplanar distance).

Table 1: Cluster size and primary particle size for PL407 and PL407/403 systems in the presence of budesonide (BUD), 2-hydroxypropyl- $\beta$-cyclodextrin $(\mathrm{HP}-\beta-\mathrm{CD})$ and the complex (CMP): budesonide-2hydroxy-propyl- $\beta$-cyclodextrin inclusion complex.

\begin{tabular}{|c|c|c|c|}
\hline $\begin{array}{c}\text { PL } \\
\text { concentration } \\
(\mathbf{\% m} / \mathbf{v})\end{array}$ & Formulations & $\begin{array}{c}\text { Cluster size }-\boldsymbol{\beta} \\
\mathbf{( n m})\end{array}$ & $\begin{array}{c}\text { Primary } \\
\text { particle size } \boldsymbol{\delta} \\
\mathbf{( n m})\end{array}$ \\
\hline & PL407 & $644.6(5)$ & $29.3(6)$ \\
\hline 18 & PL407-BUD & $652.2(6)$ & $31.1(2)$ \\
\hline & PL407-HP- $\beta-C D$ & $579.4(3)$ & $30.9(2)$ \\
\hline & PL407-CMP & $931.3(6)$ & $32.0(2)$ \\
\hline & PL407 & $835.3(1)$ & $29.6(5)$ \\
\hline 20 & PL407-BUD & $636.5(2)$ & $31.8(3)$ \\
\hline & PL407-HP- $\beta-C D$ & $556.6(3)$ & $30.9(3)$ \\
\hline & PL407-CMP & $835.9(3)$ & $31.4(3)$ \\
\hline & PL407/PL403 & $896.2(2)$ & $27.3(3)$ \\
\hline $18-2$ & $\begin{array}{c}\text { PL407/PL403- } \\
\text { HP- } \beta \text {-CD }\end{array}$ & $835.4(2)$ & $28.3(3)$ \\
\hline & $\begin{array}{c}\text { PL407/PL403- } \\
\text { CMP }\end{array}$ & $826.7(4)$ & $28.4(3)$ \\
\hline
\end{tabular}

\section{Final Considerations}

In this research, it was presented a study of the supramolecular structure of the formation of the clusters for the PL407 systems as unique or associations with other PL forming binary hydrogels PL407/PL403 with budesonide, HP- $\beta$-CD and inclusion complex budesonide-HP- $\beta-C D$, using SAXS to characterize the size of these clusters. The SAXS measurement revealed a lamellar phase organization, and using the modified Ornstein-Zernike, the form for the low q region from SAXS data, we observe changes on cluster arrangement for different systems with BUD, HP- $\beta$-CD and inclusion complex budesonide-HP- $\beta$-CD. In this study, is possible to highlight that the primary-particle size, for the PL407 18\% systems, increases when we include the cyclodextrin and the complex (displayed on Table 1). Moreover, for the highest PL407 concentration, the primary-particle size increases after CD incorporation, being also 
observed similar effects after inclusion complex addition into the hydrogels. In the case of the binary hydrogel PL407/PL403, the primary-particle size almost remains constant. These results suggest that after CDs incorporation into the hydrogels, their hydrophobic cavity can interact with the polyethylene glycol chains (PEG), suddenly increasing the primary-particle size.

On the other hand, for the inclusion complex (budesonideHP- $\beta$-CD), we have described other possible type of interaction. Considering that the hydrophobic cavity of $\mathrm{CD}$ is occupied by budesonide, this complex can possibly insert into the system amongst the PEG chains from PL, observing a similar mechanism for those two systems.

Additionally, the cluster dimension for PL407 18\% systems, decreased after CD insertion, but increased when the complex was included into the system. Those behavior was also observed for PL407 20\% the cluster decreases with the addition of CD and remains similar after inclusion complex incorporation. In the binary hydrogel system, PL407/PL403, the size of the cluster slightly decreases for both cases (with $\mathrm{CD}$ and with the complex). The reduction of the cluster size when $C D$ is added suggests that the interactions between the primary-particles are weakened due to the interaction between the hydrophobic cavity of CD and the PEGC. In the case of the inclusion of the complex, the interactions between the primary-particles remain similar, once the CD hydrophobic cavity is already filled with the drug (BUD). On the other hand, the binary system (PL407/PL403) the interaction between the particles for the systems with $\mathrm{CD}$ remains the same, suggesting that the interaction of the hydrophobic cavity of CD occurs with the PEG from PL403. Those results are of great interest for drug-delivery fields to development of new pharmaceutical formulations.

\section{Acknowledgement}

This research was supported by Fundação de Amparo à Pesquisa do Estado de São Paulo (FAPESP, Grant 2014/26200-9; 2014/14457-5), Coordenação de Aperfeiçoamento de Pessoal de Nível Superior (CAPES) and Conselho Nacional de Desenvolvimento Científico e Tecnológico (CNPq 487619/2012-9, 309612/20136). The authors are grateful to the Brazilian Synchrotron Light Laboratory for SAXS facilities (SAXS 1 beamline).

\section{References}

1. Prantera C, Marconi S (2013) Glucocorticosteroids in the treatment of inflammatory bowel disease and approaches to minimizing systemic activity. Therap Adv Gastroenterol 6: 137-156.

2. Prantera C, Scribano ML (2014) Budesonide multi-matrix system formulation for treating ulcerativecolitis. Expert Opin Pharmacother 15(6): 741-743.

3. Loftsson T, Hreinsdóttir D, Másson M (2005) Evaluation of cyclodextrin solubilization of drugs. Int J Pharm 302(1-2): 18-28.

4. Loftsson T, Brewster ME (2012) Cyclodextrins as functional excipients: methods to enhance complexation efficiency. J Pharm Sci 101(9): 30193032.

5. Kurkov S V, Loftsson T (2013) Cyclodextrins. Int J Pharm 453(1): 167180.
6. De Araújo DR, Oshiro A, Silva DC, Akkari ACS, Mello JC, et al. (2014) In: DURÁN N, GUTERRES SS, ALVEZ OL (2014) Nanotoxicology: materials, methodologies and assessments. (1 $1^{\text {st }}$ Edn.). Springer, New York, USA 1: 281-298.

7. Frisman I, Seliktar D, Bianco Peled H (2012) Nanostructuring biosynthetic hydrogels for tissue engineering: a cellular and structural analysis. ActaBiomater 8(1): 51-60.

8. Alexander S, Cosgrove T, Castle T C, Grillo I, Prescott SW (2012) Effect of Temperature, Cosolvent, and Added Drug on Pluronic-Flurbiprofen Micellization. J Phys Chem B 116(37): 11545-11551.

9. Oshiro A, da Silva DC, de Mello JC, de Moraes VW, Cavalcanti LP, et al. (2014) Pluronics f-127/l-81 binary hydrogels as drug-delivery systems: influence of physicochemical aspects on release kinetics and cytotoxicity. Langmuir 30(45): 13689-13698.

10. Chen Z, Liu Z, Qian F (2015) Crystallization of bifonazole and acetaminophen within the matrix of semicrystalline, PEO-PPO-PEO triblock copolymers. Mol Pharm 12(2): 590-599.

11. Kojarunchitt T, Baldursdottir S, Dong YD, Boyd BJ, Rades T, et al. (2015) Eur J Pharm Biopharm 89: 74-81.

12. Akkari AC, Ramos Campos EV, Keppler AF, Fraceto LF, de Paula E, et al. (2016) Colloids Surf B Biointerfaces 1(138): 138-147.

13. Kataoka T, Kidowaki M, Zhao C, Minamikawa H, Shimizu T, et al. (2006) Local and Network Structure of Thermoreversible Polyrotaxane Hydrogels Based on Poly (ethylene glycol) and Methylated $\alpha$-Cyclodextrins. J Phys Chem B 110(48): 24377-24383.

14. Kataoka T, Kidowaki M, Zhao C, Araki J, Ikehara T, et al. (2007) Thermal Properties and Microstructures of Methylated Polyrotaxane Solutions. Curr Drug Discov Technol 4(4): 275-281.

15. Bonacucina G, Spina M, Misici Falzi M, Celpi M, Pucciarelli S, et al. (2007) Effect of hydroxypropyl beta-cyclodextrin on the self-assembling and thermogelation properties of Poloxamer 407. Eur J Pharm Sci 32(2): 115-122.

16. Nogueiras Nieto L, Sobarzo Sánchez E, Gómez Amoza JL, Otero Espinar FJ (2012) Competitive displacement of drugs from cyclodextrin inclusion complex by polypseudorotaxane formation with poloxamer: implications in drug solubilization and delivery. Eur J Pharm Biopharm 80(3): 585-595.

17. Prada C, Jack KS, Grondahl L, Cooper White JJ (2013) Biomacromolecules 14(10): 3780-3792.

18. Simões SM, Veiga F, Torres Labandeira JJ, Ribeiro AC, Sandez Macho MI, et al. (2012) Eur J Pharm Biopharm 80(1): 103-112.

19. Simões SM, Veiga F, Ribeiro AC, Figueiras AR, Taboada P, et al. (2014) Eur J PharmBiopharm 87(3): 579-588.

20. Tiwari G, Tiwari R, Rai AK (2010) JPharmBioallied Sci 2: 72-79.

21. Bílkova E, Sedlák M, Dvorák B, Ventura K, Knotek P, Benes L (2010) Prednisolone- $\alpha$-cyclodextrin-star PEG polypseudorotaxanes with controlled drug delivery properties. Org Biomol Chem 23: 5423-5430.

22. Garcia Rio L, Otero Espinas FJ, Luzaro Alvarez A, Blanco Méndez J (2014) Curr Top Med Chem 14: 478-493.

23. Lazzara G, Prevost S, Gradzielski M (2011) Soft Matter 7: 6082-6609.

24. Chiappetta DA, Sosnik A (2007) Poly (ethylene oxide)-poly (propylene oxide) block copolymer micelles as drug delivery agents: improved hydrosolubility, stability and bioavailability of drugs. Eur J PharmBiopharm 66: 303-317.

25. Yamaguchi D, Mayama H, Koizumi S, Tsujii K, Hashimoto T (2008) Eur Phys J B 63: 153-163.

26. Hurd AJ, Schaefer DW, Martin JE (1987) Physical Review A 35(5): 23612364. 
ISSN: 2574-1241

DOI: 10.26717/BJSTR.2018.10.002002

Margareth KKD Franco. Biomed J Sci \& Tech Res

(C) (9) This work is licensed under Creative

Submission Link: https://biomedres.us/submit-manuscript.php

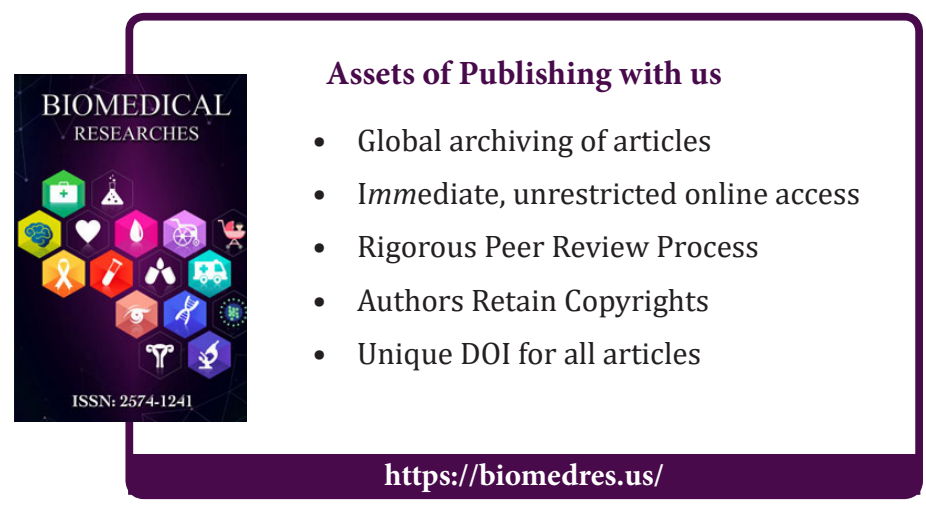

